\title{
Modeling of an optoelectronic system for data coding using orbital angular momentum of light
}

\section{Modelado de un sistema optoelectrónico para la codificación de datos usando el momento angular orbital de la luz}

\author{
J. Cuevas Cely ${ }^{1,2 *}$, C. H. Acevedo², Y. Torres Moreno ${ }^{2}$ \\ 1. Escuela de Ingeniería Eléctrica, Electrónica y Telecomunicaciones AFILIACIÓN \\ 2. GOTS, Grupo de Óptica y Tratamiento de Señales, Departamento de ciencias, Escuela de Física. AFILIACIÓN \\ ${ }^{(*)}$ E-mail: cuevasjennifer07@gmail.com
}

Received: 02/12/2016 Accepted: 07/03/2018

DOI: 10.7149/OPA.51.1.49050

\begin{abstract}
:
This paper shows the experimental and theoretical results obtained for encoding data using light beams with different values of orbital angular momentum. Light beams with orbital angular momentum were generated using holographic masks with different values of topological charge, an displayed using a transmission spatial light modulator. Furthermore, we showed the results obtained for the characterization of the spatial light modulator in the amplitude-coupled regime by using the retarder-rotor model.
\end{abstract}

Key words: Orbital angular momentum of a light, data multiplexing, spatial light modulator, retarder-rotor model.

\section{RESUMEN:}

El presente trabajo muestra los resultados experimentales y teóricos obtenidos para la codificación de datos utilizando haces luminosos con diferentes valores de momento angular orbital. Los haces luminosos con momento angular orbital fueron generados utilizando mascaras holográficas con diferentes valores de carga topológica, y desplegadas dinámicamente empleando un modulador espacial de luz por transmisión. Además se muestran los resultados obtenidos para la caracterización del modulador espacial de luz en el régimen acoplado en intensidad usando el modelo retardadorrotor.

Palabras clave: Momento angular orbital de la luz, codificación de datos, modulador especial de luz, modelo retardador rotor.

\section{REFERENCES AND LINKS / REFERENCIAS Y ENLACES}

[1] U. Efron, Spatial light modulator technology: Materials, devices, and applications (Optical science and engineering), New York, CRC Press (1994).

[2] J. Liu and J. Wang, "Polarization-insensitive PAM-4-carrying free- space orbital angular momentum (OAM) communications," Opt. Express, 24, p. 4258-4269, (2016).

[3] S. Rout and S. Sonkusale, "Wireless multi-level terahertz amplitude modulator using active metamaterial-based spatial light modulation," Opt. Express, 24, pp. 14618-14631, (2016).

[4] Y. Zhao and J. Wang, "High-base vector beam encoding/decoding for visible-light communications," Opt. Lett., 40, № 21, pp. 4843-4846, (2015).

[5] F. Sinjab y et al, "Tissue diagnosis using power-sharing multifocal Raman micro-spectroscopy and auto-fluorescence imaging," Biomed. Opt. Express, 7, pp. 2993-3006, (2016). 
[6] F. Wang et Al., "Controlled light field concentration through turbid biological membrane for phototherapy,” Biomed. Opt. Express, 6, pp. 2237-2245, (2015).

[7] P. Gao and G. Ulrich Nienhaus, "Confocal laser scanning microscopy with spatiotemporal structured illumination," Opt. Lett, 41, pp. 1193-1196, (2016).

[8] N. Matsumoto et Al., "Correction of depth-induced spherical aberration for deep observation using two-photon excitation fluorescence microscopy with spatial light modulator; Aberration-free threedimensional multiphoton imaging of neuronal activity at $\mathrm{kHz}$ rates," Biomed. and Opt. Express, 6, pp. 2575-2587, (2015).

[9] R. Kelner and J. Rosen, "Methods of Single-Channel Digital Holography for Three-Dimensional Imaging," IEEE Trans. Ind. Informatics, 12, pp. 220-230, (2015).

[10] T. Kreis, "3-D Display by Referenceless Phase Holography," IEEE Trans. Ind. Informatics, 12, pp. 685693, (2016).

[11] E. Lueder, Liquid crystal displays, New York, Jonh wiley and Sons (2010).

[12] R. Chen, Liquid crystal displays, New Jersey, Jonh wiley and Sons (2011).

[13] C. Soutar and K. Lu, "Determination of the physical properties of an arbitrary twisted-nematic liquid crystal cell," Opt. Engineering, 33, pp. 2704-2712, (1994).

[14] V. Duran, "Equivalent retarder-rotor approach to on state twisted nematic liquid crystals displays," Journ.of Apl. Physics, 99, pp. 113101-113106, (2006).

[15] C. Cuevas-Cely, C. Acevedo and Y. Torres-Moreno, "Global characterization of a nematic liquid crystal display LCX038ARA using the retarder-rotor model in the modulation amplitude regime coupled,"2017 J. Phys.: Conf. Series, 786, no N012009, pp1-4, (2017).

[16] R. M. A. Azzam and N. M. Bashara, "Ellipsometry and polarized light," Amsterdam: Elsevier, (1987).

[17] R. E. Beth, "Mechanical Detection and Measurement of the Angular Momentum of Light," Phys Rev, 50, pp. 115-125, (1936).

[18] L. Allen et Al, "Instrinsic and Extrinsic Nature of the Orbital Angular Momentum of a Light Beam," Phys. Rev. Lett, 88, pp. 3601-3605, (2002).

[19] M. J. Padgett and A. M. Yao, "Orbital angular momentum: origins, behavior and applications 3," Advances in Optics and Photonics, 2, pp. 161-204, (2012).

[20] C. H. Acevedo Cáceres, "Análisis Teórico-Experimental de un Haz con Momento Angular Orbital Entero y No Entero," Tesis de Maestría en Física, Universidad Industrial de Santander, (2012).

[21] L. Janicijevic and S. Topuzoski, "Fresnel and Franhoufer diffraction of a Gaussian laser beam by forkshaped gratings," J. Opt. Soc. Am. A, 25, pp. 2659-2669, (2008).

[22] N. R. Heckenberg et Al, "Laser beams with phase singularities," Optics and Quantum electronics, 24, pp. S951-S962, (1992).

[23] E. Santamato, "Photon orbital angular momentum: Problems and," Fortschr. Phys, 52, , pp. 11411153, (2004).

[24] C. F. Díaz, Y. Torres and C. H. Acevedo, "Superposition of two optical vortices with opposite integer or non-integer orbital angular momentum Superposición de dos vórtices óptico opuestos con momento angular orbital entero o no entero," Ingeniería e Investigación, 36 , pp. 79- 84, (2016).

[25] N. Heckenberg, R. Mcdu_ C. Smith, H. Rubinsztein and M. Wegener, "Laser beams with singularities," Opt. and Quan. Electronics, 24, S951-S962 (1992).

[25] Edmund-Optics and uEye, “USB 2.0 Cameras”, User’s manual, (2008).

\section{Introduction}

Twisted nematic spatial light modulators (TNSLMs) are optoelectronic pixilated devices that can be employed to modulate amplitude, phase or polarization of light beams in space and time [1]. Such devices are based on liquid crystal displays and they provide us novel tools to manipulate orbital angular momentum (OAM) of light in the fields of communications [2 a 4], medicine [5, 6], biology [7, 8] and holography $[9,10]$. To obtain an accurate control of the amplitude of the incident wavefront on a TNSLM and improve the experimental results obtained through them, it is important a proper calibration of the 
modulation response of a TNSLM. An appropriate amplitude calibration of a TNSLM can be achieved through the characterization of the well-known internal parameters of a liquid crystal display: molecular twist angle $(\varnothing)$, birefringence $(\beta)$ and angle molecular axis $\left(\Psi_{\mathbf{D}}\right)[11,12]$. These parameters are not providing by the manufacturer and they have slight differences between a same reference [11].

In this work we first showed experimental results of the characterization for of the amplitude response of a TNSLM based on a liquid crystal display model Sony LCX038ARA without applied voltage and with applied voltage using the Soutar-Lu method [13] and retarder-rotor method [14], respectively. Based on the amplitude characterization of our liquid crystal display model Sony LCX038ARA, we present a model of optoelectronic system to encode data using vortex beams with well-defined values of orbital angular momentum, which are produced by means of binary-amplitude computer generated holograms. In the model of optoelectronic system, we demonstrate that is possible employ the ring radii of 32 optical vortex beams with different integer OAM values to encode an alphabet. In addition, we showed the pure OAM modes generated with and without the amplitude calibration of our TNSLM.

\section{Methodology}

To model an optoelectronic system for encoding data using the orbital angular momentum of light, first we have characterized the response of our TNSLM in the amplitude-coupled regime [13] by means of the Soutar-Lu method [13] in absence of voltage and the retarder-rotor method [14] with applied voltage.

\section{2.a. Characterization of the liquid crystal matrix without applied voltage.}

To characterize the behavior of a TN-LCD without applied potential difference is necessary to carry out a process of reverse engineering to find the magnitude and orientation of the parameters of molecular twist, angle of the molecular axis and birefringence. The modulus and sign of the three parameters last mentioned have been determined using the Soutar and Lu methodology [13] with the Stokes parameters, i.e., we sandwiched our TNSLM between two polarizers to find the transmittance curves of this system at wavelength of 632.8 [nm] for the configurations: crossed polarizers and parallel polarizers, and after we must find the parameters of stokes by the procedure describing in the reference [15]. The values of birefringence and the molecular twist are necessary in the experimental characterization of the TNSLM in the on state [14].

\section{2.b. Characterization of the liquid crystal matrix with applied voltage.}

To calibration of our TNSLM with applied voltage, we have used the retarder-rotor method [14]. In this methodology the TNSLM is equivalent to a polarization system formed by a wave retarder followed by a linear polarizer, both in the presence of a potential difference applied to the TNSLM. So that, the action of a TNSLM on an incident light polarization state can simply be described by the equivalent parameters $\delta$ eq and $\emptyset$ eq, which are the retardation angle and rotation angle inserted by the TNSLM on the output light, respectively. The parameters $\delta$ eq and $\emptyset$ eq have been estimated experimentally using circularly polarized light using the schematic setup shown in figure 1. In this figure a light beam emitted by a He-Ne laser source L (wavelength $632.8[\mathrm{~nm}]$, power $5[\mathrm{~mW}]$ ) is filtered used the objective $(0)$ and the pinhole (PH). After that, the light beam is collimated using the lens L1. The emerged beam incident on our TNSLM sandwiched between the configurations: polarizer (P)-quarter wave plate 1(QWP1) and analyzer (A)quarter wave plate 2 (QWP2). The QWP1 have been placed in front of the input face of the TNSLM and rotated by 45 degrees in relation with the polarizer reference axis to generate the required circular polarization state [14]. While (QWP2) is placed after the TNSLM to measure the Stoke's parameter $\boldsymbol{S}_{3}$ [15]. Finally, the output beam converges at the image focal plane of lens L2 with the purpose to obtain efficiently intensity measurements of the beam using the power-meter (PM). The measurements of the parameters $\delta$ eq and $\emptyset$ eq were obtained experimentally for 26 images of gray levels ranging from $\mathrm{g}=0$ to $\mathrm{g}$ $=255$, which are equivalent to the applied voltage levels on our TNSLM. 


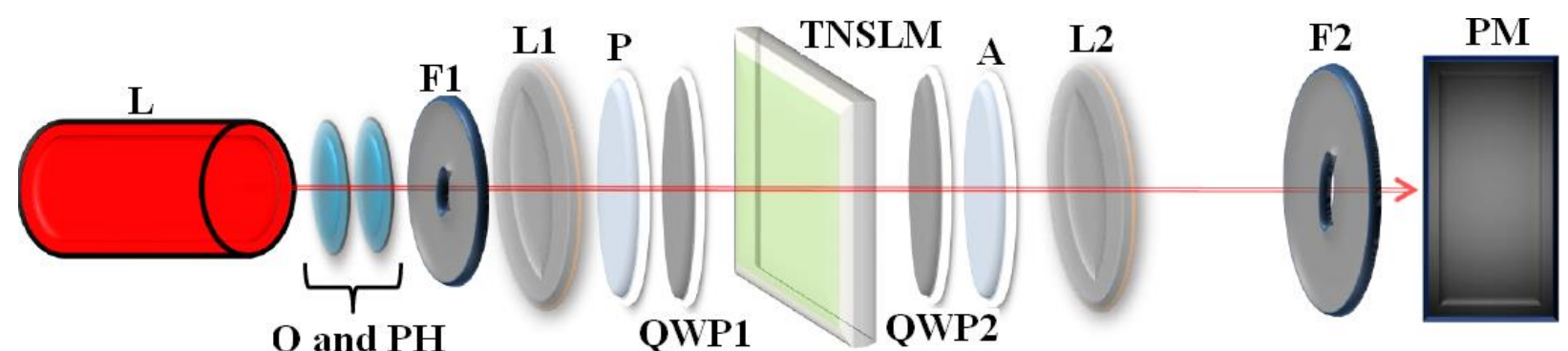

Fig. 1. Schema of experimental setup to measure the equivalent parameters $\delta$ eq and Øeq: 0: objective; PH: pinhole; F2: Pupil 1; L1: Lenses 1; P: Polarizer; QWP1: Quarter-Wave Plate 1; TNSLM: Twisted Nematic Spatial Light Modulator; A: Analyzer; QWP2: QuarterWave Plate 2; L2: Lens 2 with $f=380[\mathrm{~mm}]$ F2: Pupil 2; and PM: power meter.

Taking into account the results of the characterization in amplitude-couple regime of our TNSLM, second we have fabricated binary-amplitude computer generated holograms, which will be used to make optical vortex beams. In next subsection we describe the importance of the optical vortex beams in communications and show how we have created optical vortices to encode an alphabet.

\section{2.c. Generation encoding data of optical vortex beams.}

Optical communications are a method of transmitting information that essentially sends light signals from an emitter to a receiver [2-4]. In recent years, the OAM of a light beam has proved to be a useful property in the multiplexing of information in free space [2] and optical fibers [3], due to it allows to generate a wide range, in principle infinite spatial phase profiles with different OAM values, which are mutually orthogonal, allowing them to be multiplexed together at the same carrier frequency with independent data to increase the overall capacity of communications systems [2-4].

Optical vortex is a light beam with orbital angular momentum (OAM), i.e. is a light beam with an optical singularity created by the rotation of the wavefront of a light beam on its propagation axis [16-19]. This optical singularity in the light beam is referred to its electromagnetic field phase structure. A light beam possessing an azimuthal phase factor $\boldsymbol{e}^{\boldsymbol{i m} \boldsymbol{\theta}}$ carries a well-defined orbital angular momentum $\mathrm{m}$, where $m$ is an integer value and $\boldsymbol{\theta}$ is the azimuthal angle [18]. Figure 2 show the intensity distributions and phase profiles of Bessel-Gauss beams with OAM values $m=0, m=1, m=3$ and $m=5$, obtained numerically by using holographic masks.

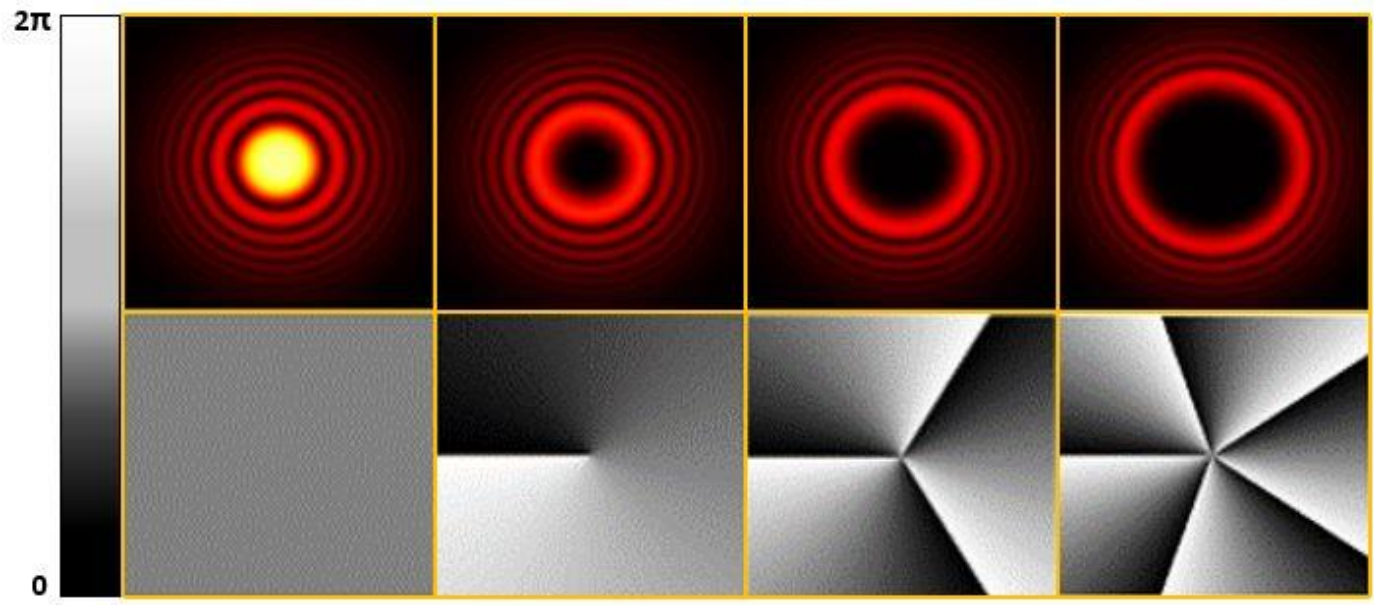

Fig. 2. (Top) simulations of the of the intensity patterns of Bessel-Gauss beams with integer OAM values of left-to-right $m=0,1,3$, and $m=5$, respectively. (Bottom) simulations of phase structures of the Bessel-Gauss beams showed above. 
In the generation of light beams with orbital angular momentum one of the most frequently used techniques is computer-generated holograms [20-23]. Theses holographic masks can be displayed on a TNSLM, which gives this method the added advantage of versatility in the in-situ manipulation of the changes make on the generated holograms [24].

Computer-generated hologram can be generated from a digital holographic pattern with forked shape, which consists of a holographic grid consisting of pixels whose transmittance function is the interference of a reference tilted plane wave and an object wave helical with the factor $\boldsymbol{e}^{i m \boldsymbol{\theta}}$, thus the interference pattern can be written as [19],

$$
I=\left|e^{i k x}+e^{i m \theta}\right|^{2}=2+2 \cos (k x-m \theta)=2(1+\cos (k x-m \theta),
$$

where $\theta=\tan ^{-1}(y / x)$ is the azimuthal angle in polar coordinates, $k$ is the wave number and $m$ is the OAM value also called the topological charge [17]. Figure 3 shows plots of $I$ for the topological charges ranging from $m=1$ to $m=6$. In this figure, one can observe by naked that the interference patterns have the wellknown forked shape, also it worth that these holograms have been created with gray levels ranging to 0 to 255.

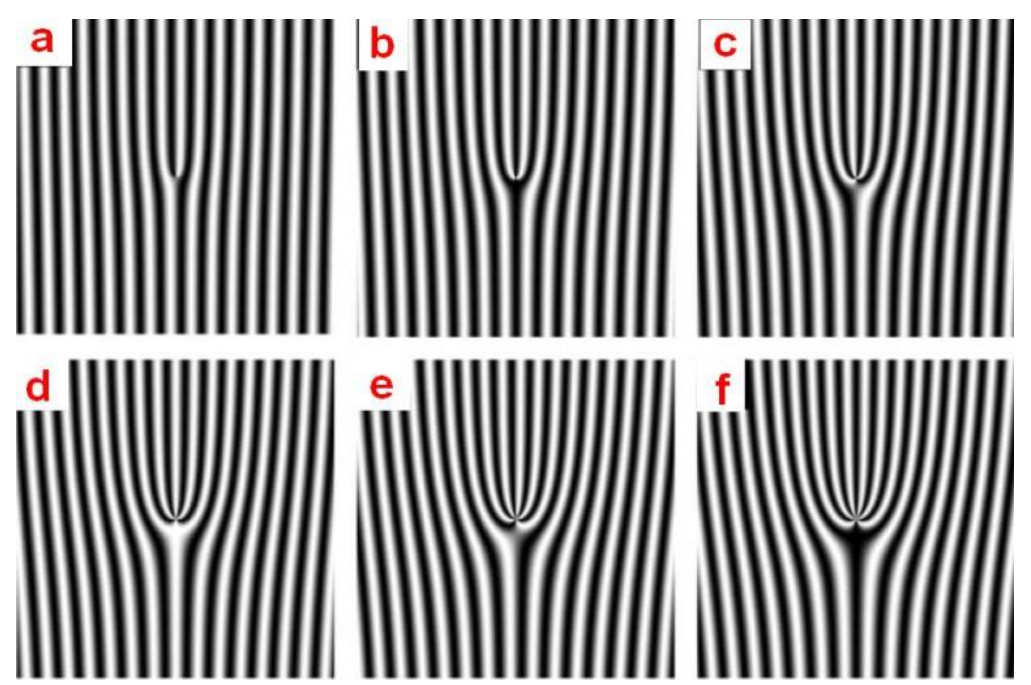

Fig.3. Plots of amplitude computer-generated holograms obtained of the Eq. (1) for the values of topological charge: a) $m=1$, b) $m=$ 2, c) $m=3$, d) $m=4$, e) $m=5$ and f) $m=6$.

\section{2.d Encoding data using optical vortex beams.}

Based on the orthogonality of the OAM modes [20] it is possible encoding information as is showed in figure 4. In this figure, a OAM phase mask with a well-defined value of OAM can easily convert a data carrying wave plane to a data-carrying OAM beam. So that, different data-carrying wave planes (or e.g Gaussian beams too) with the same electromagnetic properties such as frequency, polarization and wavelength can be encoding together due to they are indistinguishable. When different data-carrying wave plane are transformed to different OAM states, thus OAM encoding allow efficient increase the spectral efficiency and transmission capacity [3].

In our model of optoelectronic system for encoding data using the orbital angular momentum of light, we will fabricate and display binary-amplitude computer generated holograms with the minimal level gray and the two neighbor values nearest to minimum that optimize the response of our TNSLM introducing the minimal equivalent retardation values to our incident plane wavefront. 

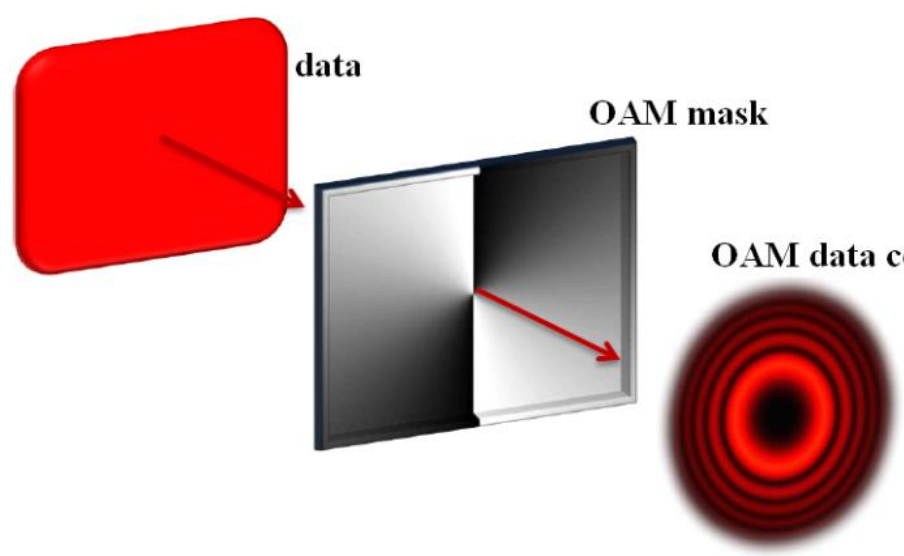

Fig. 4. Schema of conversion of data-carrying wave plane (Gaussian beam front) to a data-carrying OAM state.

\section{Results}

Table 1 shows the modulus and sign of the internal parameters: twist angle $(\varnothing)$, the angle of the molecular axis $\left(\Psi_{D}\right)$ and the birefringence $(\beta)$, when the TNSLM is in the off state, i.e., without applied voltage [15].

Table 1. Measured parameters for the TN-LCD without applied voltage.

\begin{tabular}{lc}
\hline Parameter & \\
\hline Twist angle $(\varnothing)$ & $+93.8^{\circ}$ \\
Angle of the molecular axis $\left(\Psi_{\mathrm{D}}\right)$ & $-14.9^{\circ}$ \\
Birefringence $(\beta)$ & $2.126 \mathrm{rad}$ \\
\hline
\end{tabular}

The corresponding values of the equivalent parameters $\emptyset$ eq and $\delta$ eq obtained from the experimental setup of figure 1 for the gray levels between 0 and 255 in ten-level steps are showed in figure 5 . In this figure it is possible observe the experimental data for the equivalent retardation angle (blue points) and equivalent rotation angles (black points) versus the levels of gray used. According with the experimental data, we can appreciate that the equivalent rotation angle exhibits an increasing behavior with the gray level, reaching its maximum value at $\mathrm{g}=255$. While that retardation angle show a quasi roughly symmetrical behavior between the gray levels $\mathrm{g}=30$ and $\mathrm{g}=255$ with minimal local value at $\mathrm{g}=163$, but it is close to zero for low gray levels. For this reason we can conclude that our TNSLM tends more to rotate the input polarization state than to add a phase shift on a light beam at wavelength $632.8[\mathrm{~nm}]$.

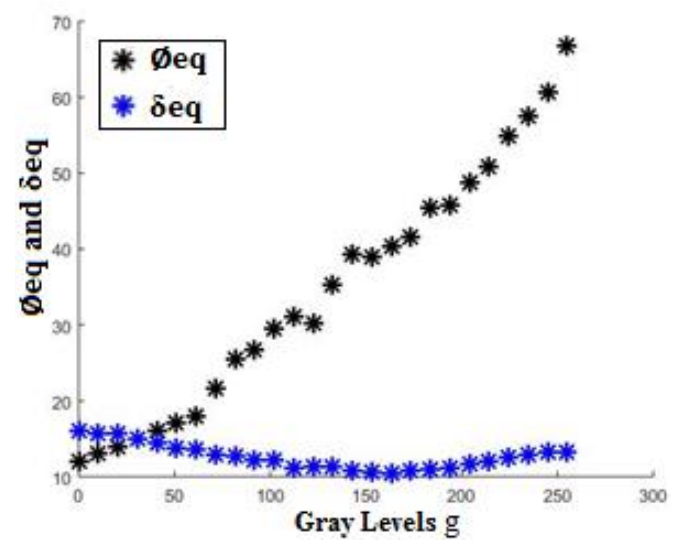

Fig. 5. Experimental results for equivalent parameters $\delta_{\mathrm{eq}}$ and $\emptyset_{\mathrm{eq}}$ as function of applied gray levels. 
By using the experimental results of characterization in the amplitude-coupled regime for our TNSLM with applied voltage, we have generated amplitude computer-generated masks with the gray levels $\mathrm{g}=$ $153, \mathrm{~g}=163$ and $\mathrm{g}=173$, which minimize the retardation angle inserted on the input beam at 632.8 [nm] in our TNSLM. The first row of Figure 6 shows three amplitude computer-generated holograms with the three gray levels last mentioned for the topological charges: $m=5, m=20$ and $m=30$; While the second row of the same figure shows the obtained results when we used the gray levels between $g=0$ and $g=255$ with equal values of topological charge.

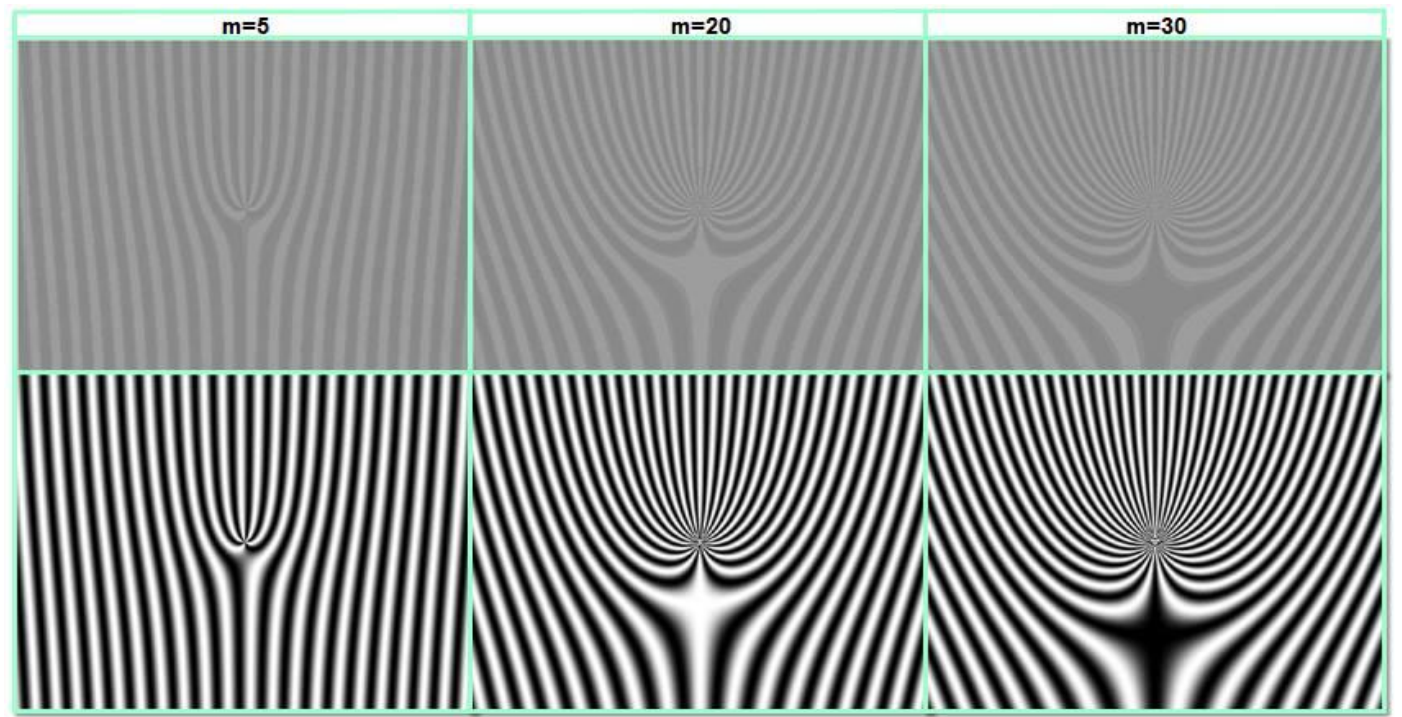

Figure 6. Plots of amplitude computer-generated holograms obtained for the mixture of gray levels $\mathrm{g}=153 \mathrm{~g}=163$ and $\mathrm{g}=173$ (top); and the 256 gray levels (bottom). In both rows were used the topological charges from left to right $m=5, m=20$ and $m=30$, respectively.

After the creation of the optimal amplitude generated-computer holograms, we have generated light beams with well-defined orbital angular momentum by displaying these masks on the TNSLM as is shown in figure 7. In this figure we showed the schema of the experimental setup used to create light beams with topological charges $m=4$ and $m=-4$ at the diffraction orders 1 and -1 respectively. In the hologram displayed on the TNSLM in figure 7 we have used all gray levels.

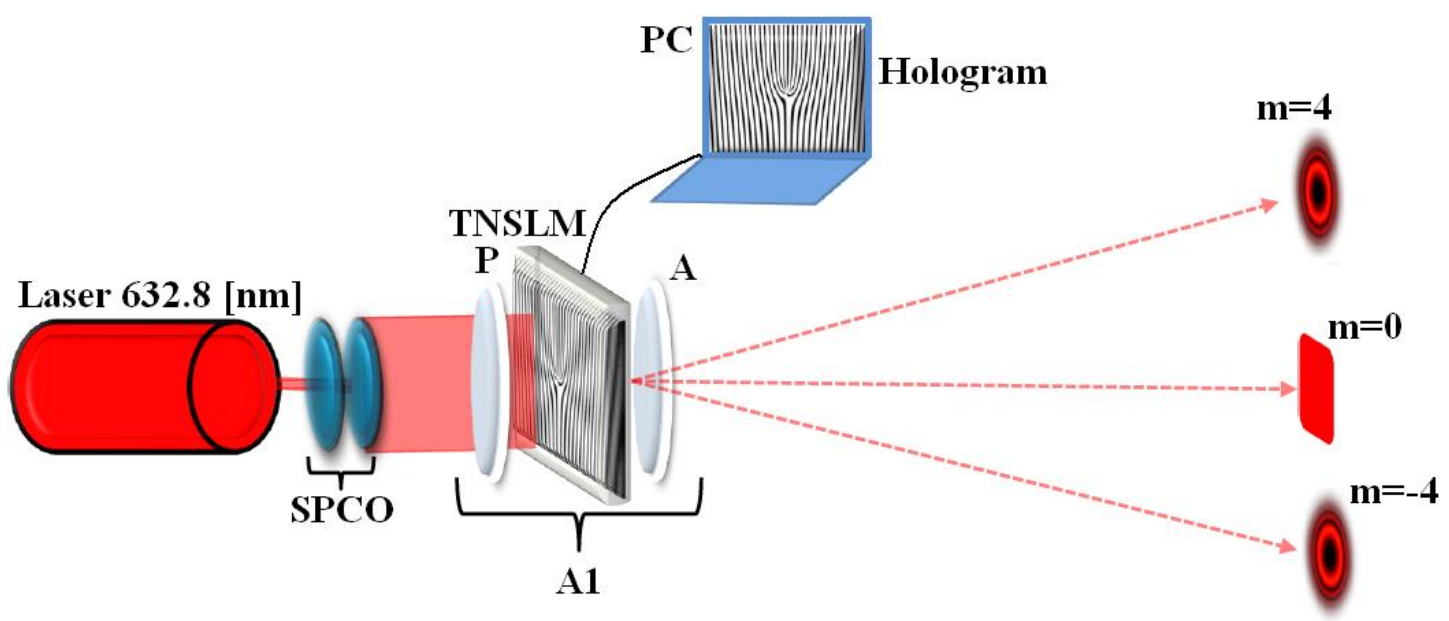

Fig. 7. Schema of experimental setup to create OAM light beams: SPCO: spatial filter and collimator; P: Polarizer; TNSLM: Twisted Nematic Spatial Light Modulator; A: Analyzer.

First row of figure 8 shows the experimental results of light beams with topological charges $m=5, m=20$ and $m=30$ at the positive first diffraction order generated by using the setup of figure 7 with the amplitude 
holographic masks showed at first row of figure 6. In this row of figure 8 we can appreciate the formation of a single quasi ring with the characteristic phase singularity of null intensity at center of light possessing $\mathrm{OAM}$, for this reason these beams can be called optical vortices [17-18]. Also we can observe that the radius of the quasi ring of each optical vortex is increased with the topological charge value. Now, using the setup of figure 7 with the amplitude holographic masks showed at second row of figure 6 , we have generated the OAM beams with topological charges $m=5, m=20$ and $m=30$ showed in the second row of figure 8 . In the OAM light beams of second row of figure 8 can see the formation of a donut with secondary rings surrounding the central ring.
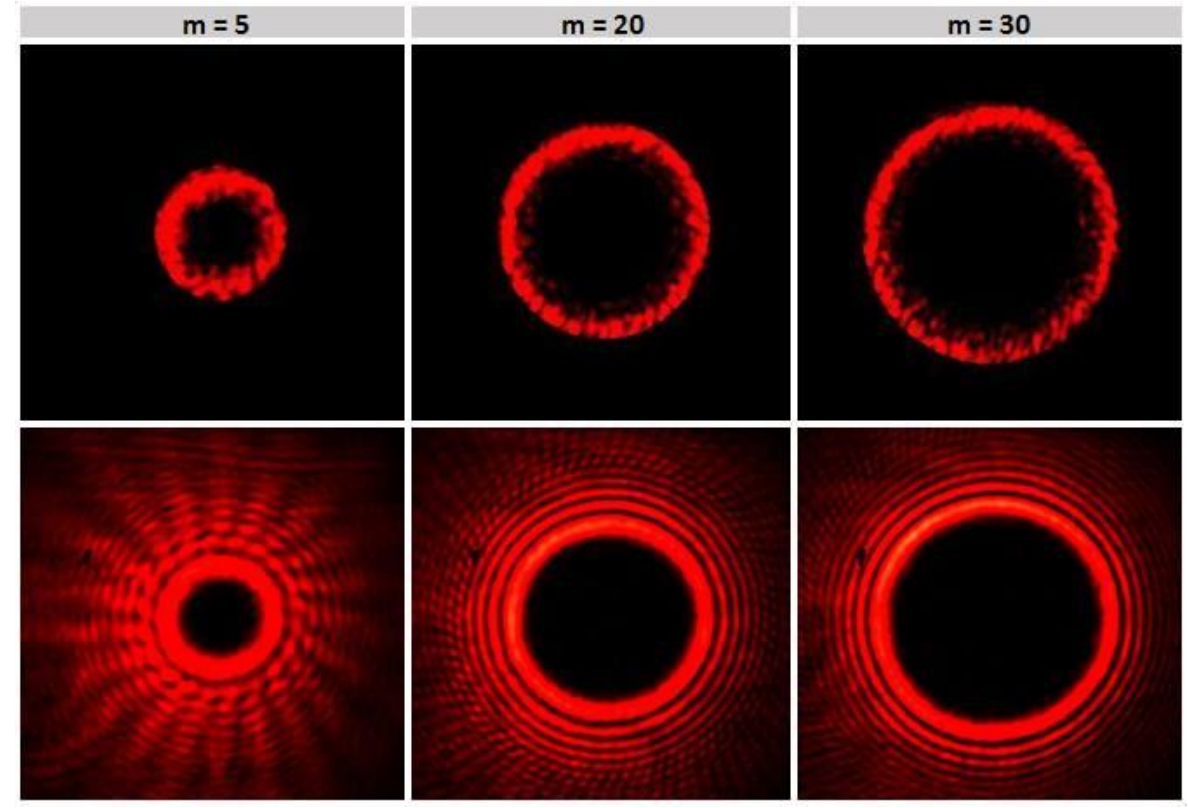

Fig. 8. OAM light beams generated with the holographic masks of the first row of figure 6 (top) and second row of figure 6 (both). In both rows the topological charges values. In both rows were used the topological charges from left to right $m=5, m=20$ and $m=30$, respectively.

Based on the generation of the OAM light beams described in the last paragraph, we proposed a free-space optoelectronic model for encoding a discrete set of data (up to five bits). We considered the average radius of each generated optical vortex as criteria of encoding of information, i.e., we have chosen the experimental optical vortices whose cross-sectional intensity profiles are the most similar to the theoretical cross-sectional intensity profiles obtained used the theorethical optical vortex with equal topological charge. To reach this last requirement, we have found the center of mass of each experimental vortex with well-defined OAM state and plotted on it an average radius in pixels (average between the internal radius and external radius) used numerical image processing. Figure 9 shows the traced average radius for an optical vortex with topological charge $m=20$.

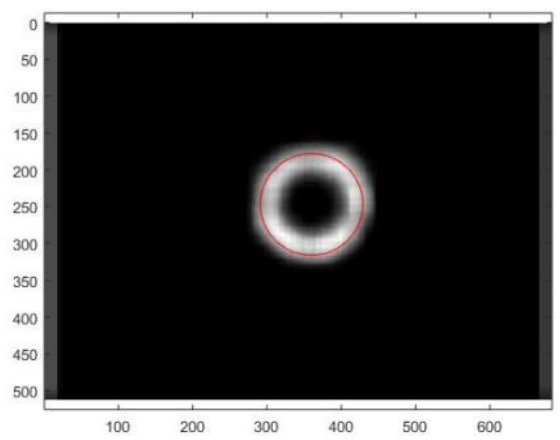

Fig. 9. Experimental average radius traced on the intensity pattern of a generated optical vortex with topological charge $\mathrm{m}=20$. 
On the other hand, the theoretical radius of each analytical vortex was found using the cross-sectional intensity profile of each vortex distribution. The left side of figure 10 shows the intensity distribution of an analytical optical vortex with topological charge $\mathrm{m}=20$, while the right side of figure 10 shows its corresponding transversal profile. In this way the theoretical radius can be calculated as the number of pixels from the center to either of the two lateral maximums. It is worth noting that in both average radii were calculated used matrices with equal dimensions in the vertical and horizontal directions.

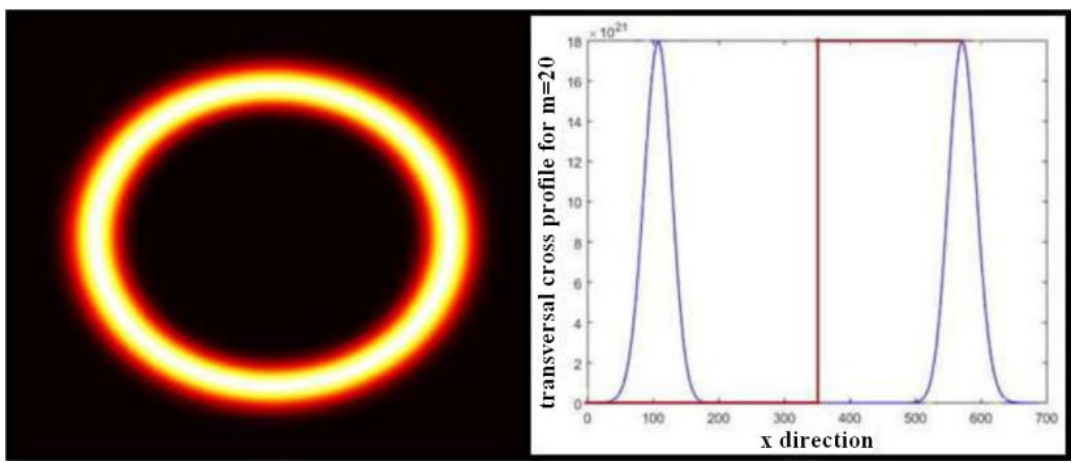

Fig. 10. Theoretical average radius traced on the intensity pattern of an optical vortex with topological charge m=20.

Columns 2 and 3 of Table 2 show theoretical and experimental average radius values in pixels calculated using the procedures before described for the topological charges ranging from $m=0$ to $m=40$, respectively. Column 4 of this table includes the absolute error. It possible to note that this error does not exceed $11 \%$ for the topological charges between $m=17$ and $m=40$. For this reason, we have chosen the 23 optical vortices between $m=17$ and $m=40$ as a reliable basis for data coding. However, in order not to waste data between $m=0$ and $m=16$, have been chosen only the odd values as complement of the basis values ranging from $m=17$ and $m=40$. According with last criteria the total number of optical vortices for encoding data is 32 .

Table 2. Results of the average experimental and theorethical radii.

\begin{tabular}{|c|c|c|c|}
\hline \multirow{2}{*}{ CHARGE } & $\begin{array}{c}\text { THEORETICAL } \\
\text { RADIUS (pixels) }\end{array}$ & $\begin{array}{c}\text { EXPERIMENTAL } \\
\text { RADIUS (pixels) }\end{array}$ & ABSOLUTE ERROR \\
\hline 40 & 91,12 & 90 & 1,23 \\
\hline 39 & 89,76 & 88 & 1,96 \\
\hline 38 & 88,74 & 87 & 1,58 \\
\hline 37 & 87,38 & 86 & 1,57 \\
\hline 36 & 86,36 & 85 & 1,57 \\
\hline 35 & 85,34 & 84 & 0,75 \\
\hline 34 & 83,98 & 82 & 0,33 \\
\hline 33 & 82,62 & 81 & 0,13 \\
\hline 32 & 81,26 & 80 & 0,15 \\
\hline 31 & 79,90 & 79 & 0,62 \\
\hline 30 & 78,88 & 78 & \\
\hline 29 & 77,52 & & 167 \\
\hline
\end{tabular}




\begin{tabular}{|c|c|c|c|}
\hline 28 & 76,16 & 77 & 1,10 \\
\hline 27 & 74,80 & 76 & 1,60 \\
\hline 26 & 73,44 & 75 & 2,12 \\
\hline 25 & 72,08 & 74 & 2,66 \\
\hline 24 & 70,38 & 73 & 3,72 \\
\hline 23 & 69,36 & 72 & 3,81 \\
\hline 22 & 67,66 & 71 & 4,94 \\
\hline 21 & 65,96 & 70 & 6,12 \\
\hline 20 & 64,26 & 69 & 7,38 \\
\hline 19 & 62,56 & 68 & 8,70 \\
\hline 18 & 61,20 & 67 & 9,48 \\
\hline 17 & 59,50 & 66 & 10,92 \\
\hline 16 & 57,46 & 65 & 13,12 \\
\hline 15 & 55,76 & 64 & 14,78 \\
\hline 14 & 53,72 & 63 & 17,27 \\
\hline 13 & 51,68 & 62 & 19,97 \\
\hline 12 & 49,64 & 61 & 22,88 \\
\hline 11 & 47,60 & 60 & 26,05 \\
\hline 10 & 45,56 & 59 & 29,50 \\
\hline 9 & 43,18 & 58 & 34,32 \\
\hline 8 & 40,80 & 57 & 39,71 \\
\hline 7 & 38,08 & 56 & 47,06 \\
\hline 6 & 35,36 & 55 & 55,54 \\
\hline 5 & 32,30 & 54 & 67,18 \\
\hline 4 & 28,90 & 53 & 83,39 \\
\hline 3 & 25,16 & 52 & 106,68 \\
\hline 2 & 20,40 & 51 & 150,00 \\
\hline 1 & 14,28 & 50 & 250,14 \\
\hline 0 & 43,87 & 48 & 9,41 \\
\hline
\end{tabular}

Table 3 shows the 32 optical vortices with the well-defined values of topological charge and a simple binary algorithm of 5 bits coding $\left(2^{\wedge} 5=32\right)$ created with them. In this table we have assigned a letter of the alphabet to the selected OAM modes.

Figure 11 shows the structure of the model of proposed encoder. In this figure can be observed as a plane wavefront illuminates different holograms displayed on our TN-LCD with the coded letters of the alphabet selected for the message. The TN-LCD has a transition time of the holograms of approximately 1/60 [s], it is means that it is possible send a coded letter each $1 / 60$ [s]. The generated optical vortex with the coded letter is propagating in the free space (channel) and it is filter through the open aperture F1. The intensity distribution is detected by a monochrome CCD camera [25] placed at 38.5 [cm] from the TNSLM. It deserves special mention that, to make sure the total propagation length is about $38.5[\mathrm{~cm}]$, which is the maximum distance to detect the optical vortex with the coded letter $A$ within the CCD camera sensor 
connected to computer PC2. In this way, it is possible send successively coded letters and acquired the intensity patterns on the CCD camera. Finally, a computer with installed software such as Matlab allows storing the experimental optical vortex images with a recording time of 1/30 [s] to be discriminated by its radii according to the OAM values. It is worth noting that the proposed decoder in this work is static, i.e., it necessary to store all images to be later processed and discriminated according to the OAM states with the encoding information.

Table 3. Assigned alphabet

\begin{tabular}{|c|c|c|c|}
\hline ASSIGNED ALPHABET & MODE 1 & ASSIGNED ALPHABET & MODE 1 \\
\hline A & 40 & $\tilde{\mathrm{N}}$ & 26 \\
\hline B & 39 & 0 & 25 \\
\hline $\mathrm{C}$ & 38 & $\mathrm{P}$ & 24 \\
\hline D & 37 & $Q$ & 23 \\
\hline$E$ & 36 & $\mathrm{R}$ & 22 \\
\hline $\mathrm{F}$ & 35 & $S$ & 21 \\
\hline $\mathrm{G}$ & 34 & $\mathrm{~T}$ & 20 \\
\hline $\mathrm{H}$ & 33 & $\mathrm{U}$ & 19 \\
\hline I & 32 & $\mathrm{~V}$ & 18 \\
\hline $\mathrm{J}$ & 31 & $\mathrm{~W}$ & 15 \\
\hline $\mathrm{K}$ & 30 & $\mathrm{X}$ & 11 \\
\hline $\mathrm{L}$ & 29 & $\mathrm{Y}$ & 7 \\
\hline$M$ & 28 & $\mathrm{Z}$ & 3 \\
\hline $\mathrm{N}$ & 27 & - & - \\
\hline
\end{tabular}
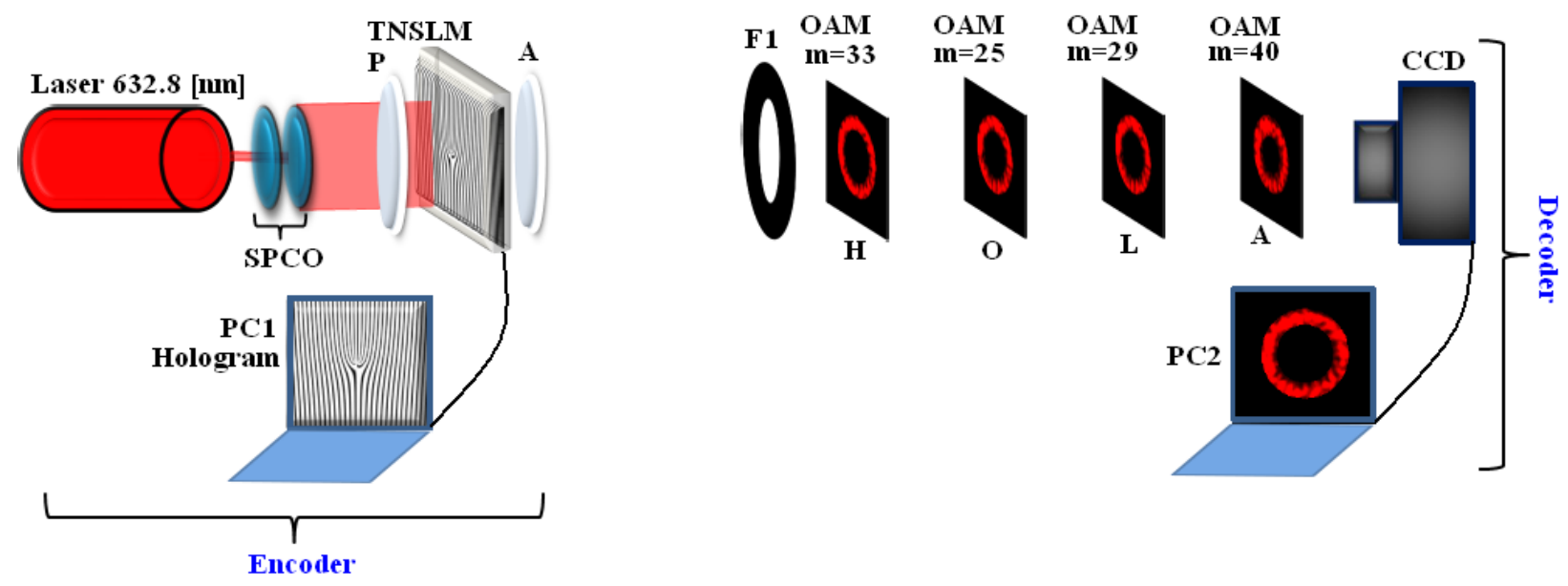

Fig. 11. Schema of the model of the proposed experimental setup for OAM encoding and decoding. 


\section{Conclusions}

For coding information on experimental optical vortices was established as geometric criteria, those whose cross-sectional intensity profile was the most similar with $11 \%$ of reliability equal or less, to that obtained for the same theoretical optical vortex with the same topological load what Allows generating 32 orbital angular moments of interest required to encode a 5-bit data.

\section{Acknowledgements}

Research partially supported by Universidad Industrial de Santander with the projects 5708 and 1788 . Thanks to Colciencias for financial support to project 110256934957: "Dispositivos ópticos para sistemas de alta dimensionalidad QKD (Quantum Key Distribution) basados en el momento angular orbital de la luz". C.H. Acevedo Cáceres acknowledges the support and sponsorship from Call 647-2015: National Doctoral program of Colciencias. 